\title{
Opto-thermal Radiometry for Detection of Surface Contamination
}

\section{Zhang ${ }^{1,2} \cdot$ C. Bontozoglou ${ }^{1} \cdot$ P. Xiao ${ }^{1}$}

Received: 12 September 2019 / Accepted: 28 January 2020 / Published online: 20 February 2020 (c) The Author(s) 2020

\begin{abstract}
The aim of this work is to conduct a proof-of-concept study on detection of surface contamination by biological and chemical agents using opto-thermal transient emission radiometry (OTTER). OTTER is an infrared remote sensing technology that has been developed in our research group for skin measurements. It is based on the measurement of laser-induced transient thermal emission spectra in the infrared fingerprint region, and has advantages of non-contact, non-invasive, spectroscopic in nature, and can work on arbitrary surfaces of any materials. In this paper, two types of experiments were conducted, different ingredients on the surface of a sample material and different ingredients on the skin surface. The results show that by measuring the OTTER signal at different detection wavelengths, e.g., $6.05 \mu \mathrm{m}$, $6.48 \mu \mathrm{m}, 7.79 \mu \mathrm{m}, 8.5 \mu \mathrm{m}, 9.5 \mu \mathrm{m}, 11.5 \mu \mathrm{m}, 13.1 \mu \mathrm{m}$, it is possible to differentiate from skin and skin with different ingredients on the surface. The next step is to develop mathematical models and analytical algorithms to quantify the ingredient concentration on the surface.
\end{abstract}

Keywords Biological and chemical agents · Infrared fingerprint region · Laser · Opto-thermal radiometry $\cdot$ Surface contamination detection

This article is part of the selected papers presented at the 20th International Conference on Photoacoustic and Photothermal Phenomena.

P. Xiao

perry.xiao@1sbu.ac.uk

1 School of Engineering, London South Bank University, 103 Borough Road, London SE1 0AA, UK

2 Auckland Tongji Medical \& Rehabilitation Equipment Research Centre, Tongji Zhejiang College, Jiaxing, China 


\section{Introduction}

Surface contamination detection is very important in many scenarios, such as food safety, public place security, e.g., stadiums, stations, and airports. To be able to detect biological and chemical agents on the surface of goods and personal is also important during the chemical warfare. A non-contact, non-invasive technique will be ideal for such applications. Researchers from US Naval Research Laboratory have developed a technique called Photothermal IR imaging spectroscopy (PT-IRIS) for rapid standoff detection of trace of explosives [1-3]. It uses a tunable quantum cascade laser (QCL, $5 \mu \mathrm{m}$ to $8 \mu \mathrm{m}$ ) to heat up a surface of interest, and uses a thermal camera $(8 \mu \mathrm{m}$ to $12 \mu \mathrm{m})$ to view thermal contrast. They have demonstrated the principle by using the explosives trinitrotoluene (TNT), cyclotrimethylenetrinitramine (known as RDX), ammonium nitrate (NH4NO3, an explosive precursor), or sugar (sucrose, a confusant or an explosive fuel) on transparent, absorbing, and reflecting substrates. Trace explosives from particles as small as $10 \mu \mathrm{m}$ have been detected.

Rasskazov et al. [4] have developed a non-contact no-reagents system operating in the eye-safe $1560 \mathrm{~nm}$ to $1800 \mathrm{~nm}$ wavelength range for standoff trace detection of explosives. It uses a fiber laser at $1546 \mathrm{~nm}$ central wavelength (12 $\mathrm{nm}$ at fullwidth half-maximum, modulated to sub-500 fs pulses at a $2 \mathrm{MHz}$ repetition rate) to scan the sample surface, and a fast photodiode to collect the scattered light. The results showed that it is possible to detect a number of chemicals including explosives on a variety of surfaces at sub-microgram per $\mathrm{cm}$ concentration.

Dupuis et al. [5] have developed a quantum-cascade-laser-based detector for proximal screening of surface contaminants including explosives, chemical warfare agents (CWAs), and toxic industrial chemicals and materials. It uses a spectrally tunable external cavity (EC) QCL to illuminate the sample, and a FLIR Tau 336 thermal camera $(336 \times 256$ pixels, $7.5 \mathrm{~Hz}, 7.5 \mu \mathrm{m}$ to $13 \mu \mathrm{m})$ to detect the sample's thermal radiation. By tuning the QCL with different wavelengths, a stack of spectral thermal images can be obtained. By analyzing the images, a number of samples have been studied. This technology has been demonstrated for high-throughput security screening of people and vehicles.

Farahi et al. [6] have developed a pump-probe photothermal spectroscopy technique to obtaining compositional information for objects from a distance. They used tunable QCLs $(9.26 \mu \mathrm{m}$ to $9.8 \mu \mathrm{m}, 0.2 \mathrm{kHz}, 0.02 \mu$ s pulse width) to excite the molecules in the mid-infrared region, and used a HeNe laser ( $5 \mathrm{~mW}$ in power, $632.8 \mathrm{~nm}$ in wavelength) as a pump-probe light. By measuring the variation of the photothermal process of the substances, they can get spectral fingerprints of substances from a variable standoff distance.

In this paper, we present our latest research on surface contamination detection by using Opto-thermal transient emission radiometry (OTTER) [7-12]. Apart from being non-contact, non-invasive and spectroscopic in nature, the advantages of using OTTER for surface contamination detection are as follows: it can work on any arbitrary unprepared sample surfaces, it is simple to make a measurement (point and shoot), and with the advances of modern optical and 
electronic technologies, it is possible to make a small, portable, low-cost measurement device. We will first present the theoretical background, and then show the experimental results.

\section{Methodology and Materials}

The apparatus used in this study are conventional Opto-thermal transient emission radiometry (OTTER). The details of OTTER can be found elsewhere [7, 8]. It essentially uses a pulsed laser (Er:YAG laser, $2.94 \mu \mathrm{m}, 100$ ns pulse duration, $1 \mathrm{~mm}$ diameter laser spot size, a few mille joules per pulse) as a heat source to heat the sample, and a fast infrared MCT detector (mercury cadmium telluride, with spectrum responsive range: $3 \mu \mathrm{m}$ to $15 \mu \mathrm{m}, \mathrm{FOV}=60^{\circ}$, Infrared Associates Inc, USA) to measure the sample's consequent blackbody radiation increase. By analyzing the shape of the signals, we can get optical properties, thermal properties, and layered structure information of the sample. By using different narrow bandpass mid-infrared interference filters in front of the MCT detector, we can also select different detection wavelengths. By plotting the data at different detection wavelengths, we can get an OTTER spectrum. With the OTTER spectra, it is possible to detect surface contaminations, based on different absorptions of molecules at the fingerprint mid-infrared spectra range, i.e., $3 \mu \mathrm{m}$ to $15 \mu \mathrm{m}$.

The OTTER signal can be generally expressed as [7-9]

$$
S(t)=A \mathrm{e}^{t / \tau} \operatorname{erfc} \sqrt{t / \tau},
$$

where $A$ is the amplitude of the signal, $\tau=\frac{1}{\beta^{2} D}$ is the signal decay lifetime, $\beta$ is the sample's emission absorption coefficient, and $D$ is the sample's thermal diffusivity. By fitting the OTTER signal using Eq. 1, we can get the best fit $\beta$. Different molecules have different $\beta$ values at different wavelengths. Therefore by measuring the samples at different wavelengths, it is possible to figure out what molecules present on the sample surface.

Figure 1 shows the steps:

1. Perform the measurements on samples by using OTTER at different detection wavelengths, e.g., $6.05 \mu \mathrm{m}, 6.48 \mu \mathrm{m}, 7.79 \mu \mathrm{m}, 8.5 \mu \mathrm{m}, 9.5 \mu \mathrm{m}, 11.5 \mu \mathrm{m}, 13.1 \mu \mathrm{m}$, see Fig. 1a.

2. Plot the OTTER signals at different detection wavelengths, see Fig. 1b.

3. For each OTTER signal at each detection wavelength, analyze the whole signal curve using traditional least squares fitting with Eq. 1 [7, 8] to get an average absorption coefficient, see Fig. 1c. In this approach, we use the following equation to calculate the Chi square $\left(\chi^{2}\right)$, and continuously adjust the values of $A$ and $\tau$ in Eq. (1) in order to get the minimum Chi square $\left(\chi^{2}\right)$. Here $y\left(t_{i}\right)$ is the OTTER measurement signal data at time $t_{i}$, and $S\left(t_{i}\right)$ is the Eq. 1 value at time $\mathrm{t}_{\mathrm{i}} . \mathrm{N}$ is the total data points. 


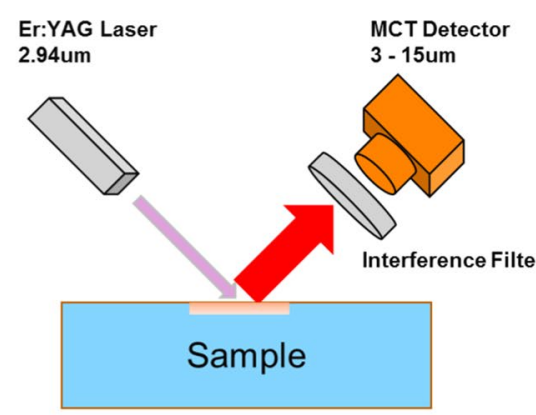

(A)
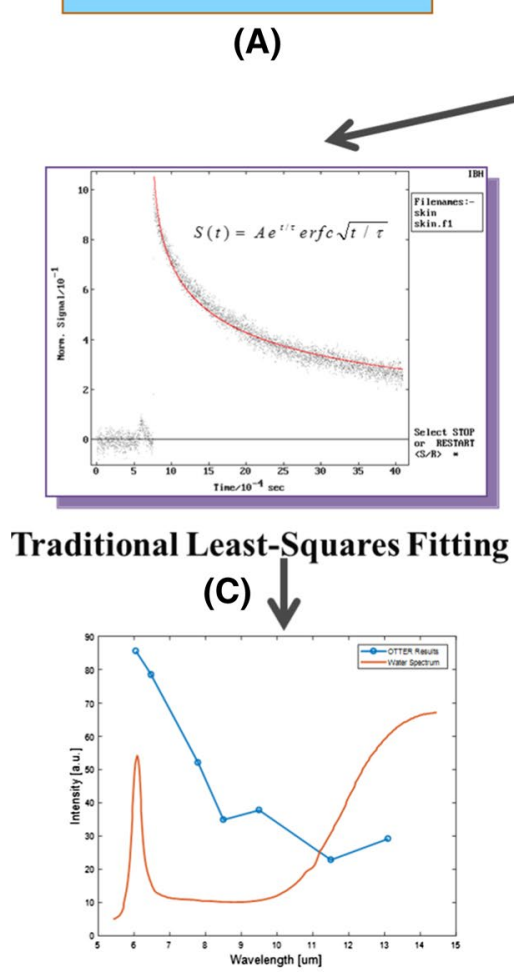

(D)

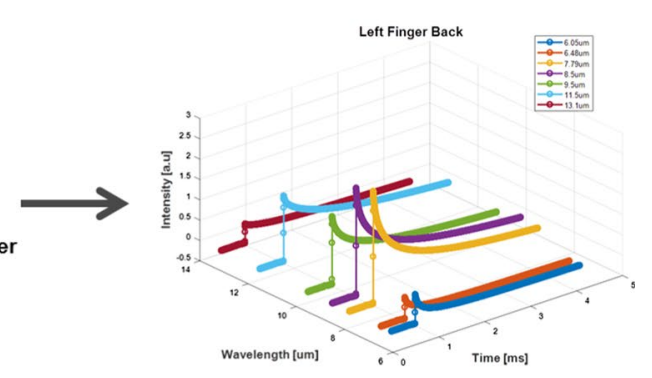

(B)
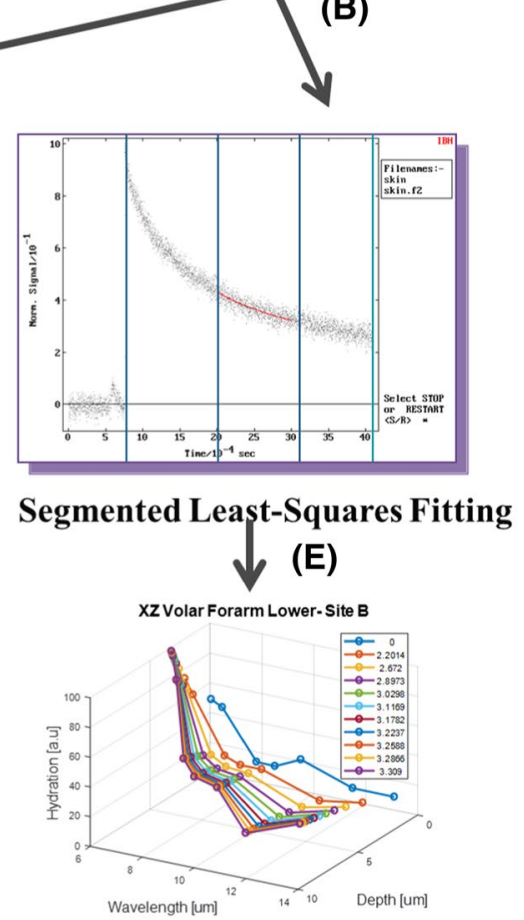

(F)

Fig. 1 The schematic diagram of OTTER measurements (a), the OTTER signal at different detection wavelengths, e.g., $6.05 \mu \mathrm{m}, 6.48 \mu \mathrm{m}, 7.79 \mu \mathrm{m}, 8.5 \mu \mathrm{m}, 9.5 \mu \mathrm{m}, 11.5 \mu \mathrm{m}, 13.1 \mu \mathrm{m}$ (b), analyzing the OTTER signal using traditional least squares fitting (c), to get a single OTTER spectrum compared with water spectrum (d), analyzing the OTTER signal using segmented least squares fitting (e), to get OTTER depth-resolved spectra (f)

$$
\chi^{2}=\sum_{i=1}^{N} \frac{\left(y\left(t_{i}\right)-S\left(t_{i}\right)\right)^{2}}{N-1}
$$

4. Plot OTTER results at all detection wavelengths together to create an OTTER spectrum, see Fig. 1 d. 
5. Instead of analyzing the whole signal curve, as in step 3, we can also cut the signal curves into pieces, and analyze each piece separately by using segmented least squares fitting with Eq. 1 [10], see Fig. 1e.

6. The result from each piece of signal represents the information at different sample depths [10]; by plotting OTTER results of different pieces, at all detection wavelengths together, we can get OTTER spectra at different depths of the sample, i.e., depth-resolved spectra, see Fig. 1f.

OTTER spectra can be used to analyze the sample surface chemical components, and OTTER depth-resolved spectra can also analyze the sample's chemical components beneath the surface.

Materials used in study are all standard materials, e.g., tissue paper (Marks \& Spencer, UK), extra virgin olive oil (Marks \& Spencer, UK), and MS Neat (methyl salicylate) (Sigma-Aldrich, UK).

\section{Results and Discussions}

\subsection{Different Chemicals on a Sample Surface}

In this experiment, the presence of MS Neat (methyl salicylate) and extra virgin olive oil on a tissue paper are studied. First, using Step 1, as described above, OTTER signals were obtains for tissue only and MS Neat only. Extra virgin olive oil does not absorb Er:YAG laser $2.94 \mu \mathrm{m}$ light and hence has no signal on its own. Also obtained were OTTER signals for tissue with MS Neat, tissue with extra virgin olive oil, and tissue with MS Neat and extra virgin olive oil. Figure 2 shows the typical OTTER signals at different detection wavelengths, and in this case, it is MS Neat. Other OTTER signals are different but similar. Second, by using calculations as shown in the above Steps 3 and 4, we can calculate OTTER spectra. Figure 3 shows the calculated OTTER spectra of MS Neat only, tissue only, MS Neat and tissue, olive oil and tissue, as well as MS Neat, olive oil, and tissue all together, compared with water spectrum (not to scale). The results show that different chemicals and different combinations of chemicals produce different spectra; therefore, it is possible to identify different chemicals from one spectrum.

\subsection{Different Chemicals on Skin Surface}

In this experiment, the presence of MS Neat (methyl salicylate) and extra virgin olive oil on an in vivo finger skin are studied. First, using Step 1, as described above, the OTTER signals were obtained for finger skin only, MS Neat and finger, extra virgin olive oil and finger, and MS Neat, extra virgin olive oil, and finger. Figure 4 shows the comparison of OTTER signals at different detection wavelengths, for finger skin only and for finger skin with MS Neat. There are obvious changes in the amplitudes and the shapes of the signals due to the presence of MS Neat on finger. Second, by using calculations as shown in the above Steps 3 and 4, we can get 


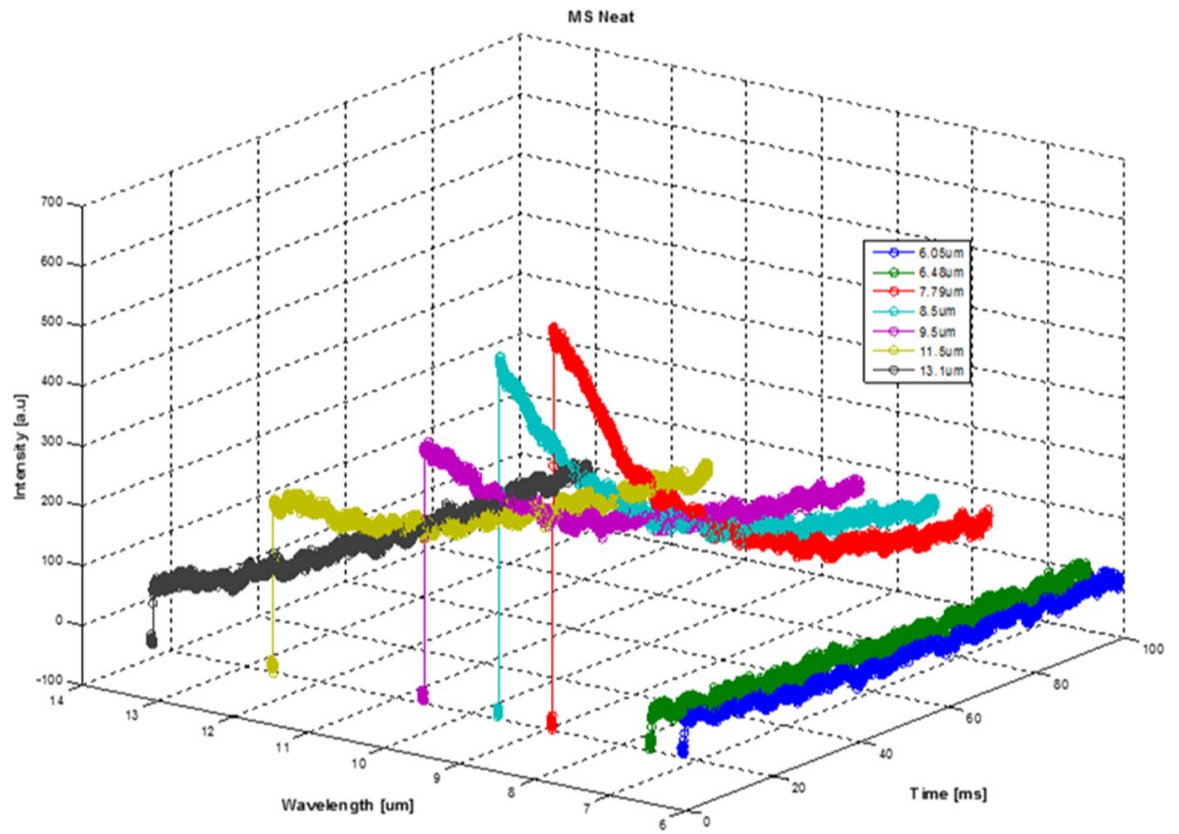

Fig. 2 A typical OTTER signal (MS Neat) at different detection wavelengths

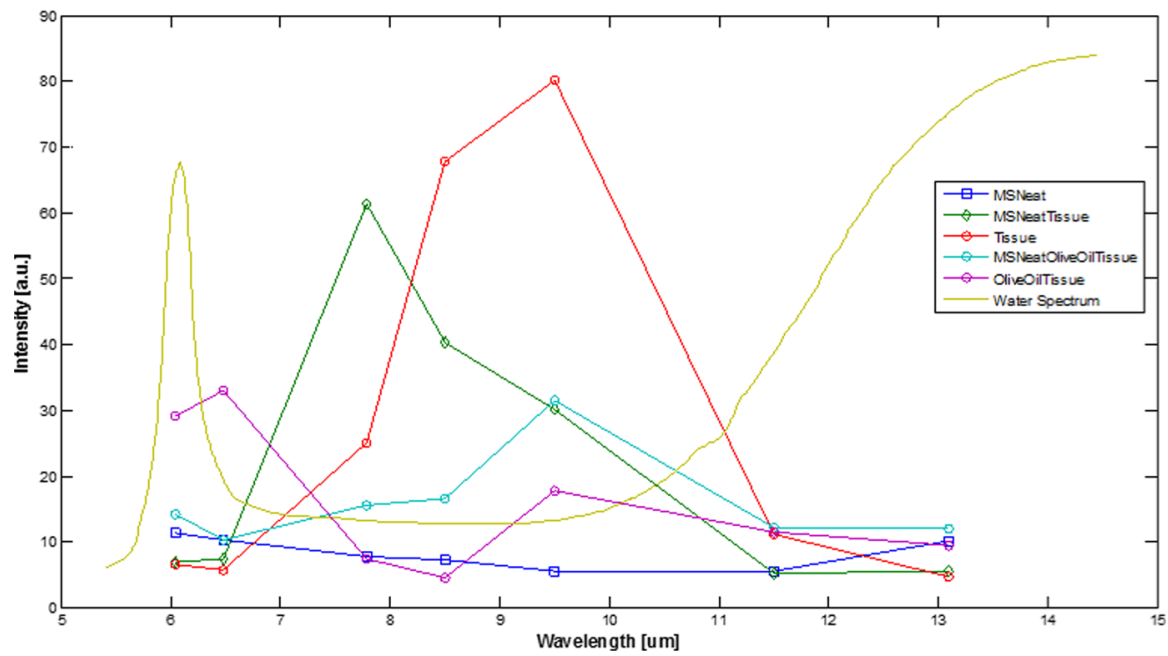

Fig. 3 The OTTER spectra of MS Neat only, olive oil only, tissue only, MS Neat and tissue, olive oil and tissue, as well as MS Neat, olive oil, and tissue all together, compared with water spectrum (not to scale)

OTTER spectra. Figure 5 shows the OTTER spectra of MS Neat only, olive oil only, finger skin only, MS Neat and finger skin, olive oil and finger skin, as well as MS Neat, olive oil, and skin all together, compared with water spectrum (not to scale). 

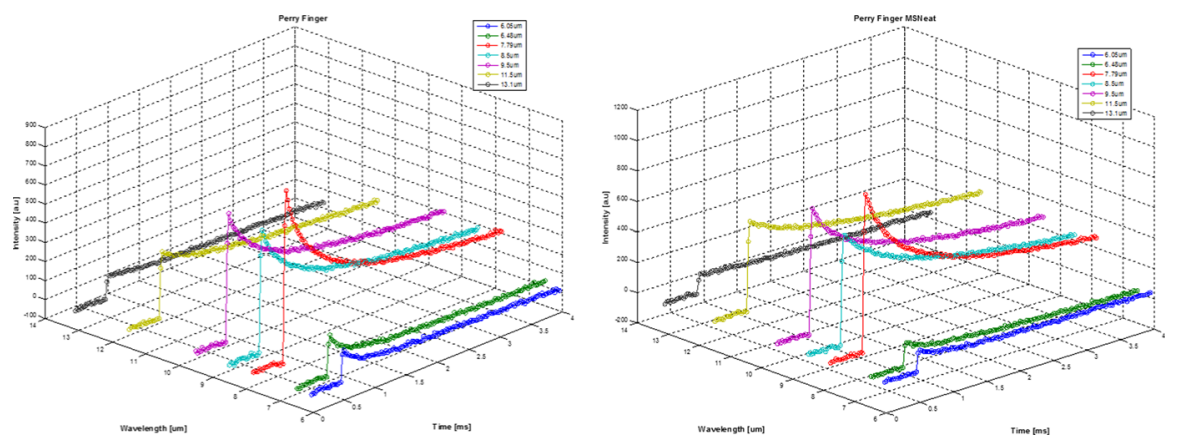

Fig. 4 The OTTER signals at different detection wavelengths for finger skin (left) and MS Neat and finger skin (right)

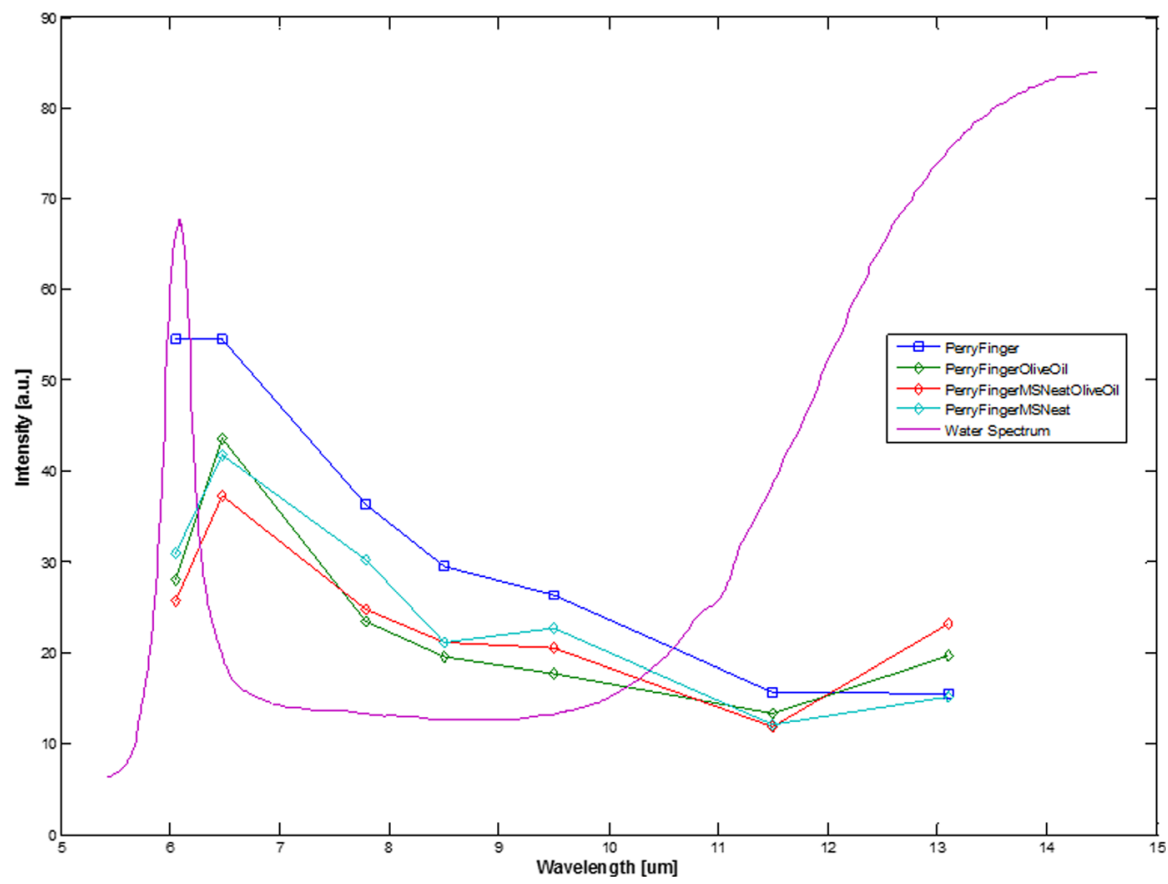

Fig. 5 The OTTER spectra of MS Neat only, olive oil only, finger skin only, MS Neat and finger skin, olive oil and finger skin, as well as MS Neat, olive oil, and finger skin all together, compared with water spectrum (not to scale)

The results again show that different chemicals and different combinations of chemicals produce different spectra; therefore, it is possible to identify different chemicals present on the skin surface.

By analyzing the OTTER signals at different wavelengths (as shown Fig. 4) using Steps 5 and 6, as illustrated above, we can also get an depth-resolved OTTER 

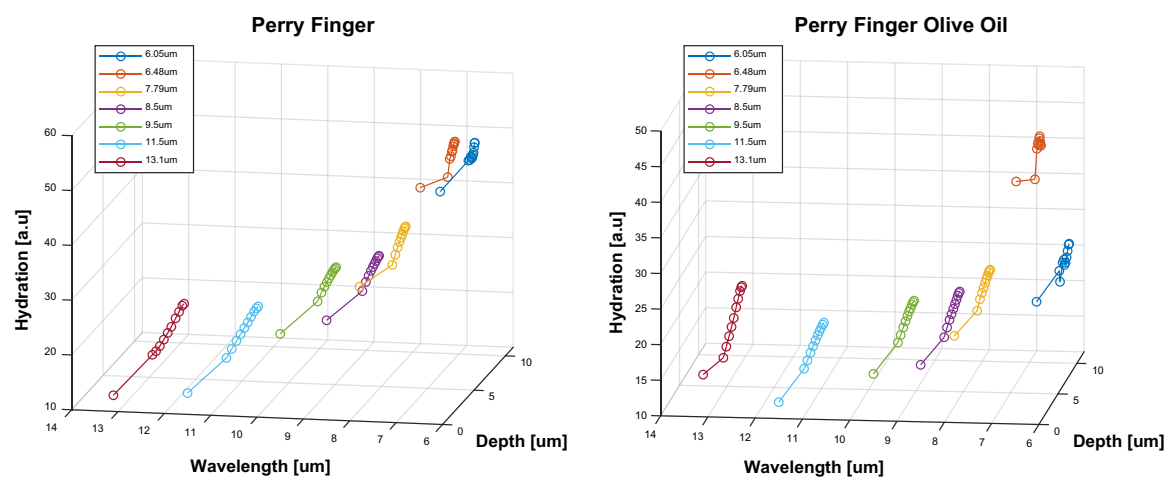

Fig. 6 The depth-resolved OTTER spectra of finger skin only (left), as well as olive oil and finger skin (right)

spectra, i.e., OTTER spectra at different depths within skin, see Fig. 6. We can see that deeper skin has higher values at all wavelengths, which means there is a positive gradient toward deeper skin. Once olive oil is applied on skin, it also changes the absorptions at different wavelengths, but the largest change is at $6.05 \mu \mathrm{m}$. The results show that OTTER can see about $10 \mu \mathrm{m}$ into skin with about $1 \mu \mathrm{m}$ depth resolution. No other techniques can achieve this.

\section{Conclusions}

The results show that OTTER (opto-thermal transient emission radiometry) is a promising technology that can be used for surface contamination detection measurements. By performing the measurements at different wavelengths, we can get an OTTER spectrum. OTTER spectrum can show the differences of multiple chemicals present on the sample surface. By analyzing the OTTER signal using segmented least squares fitting, we can also get a depth-resolved OTTER spectrum. The depthresolved OTTER spectrum not only can show the sample surface chemicals, but also can show the chemicals beneath the surface. The next step is to develop mathematical models and analytical algorithms to quantify the ingredient concentration on the surface.

Acknowledgement We thank London South Bank University for the financial support. We also thank Biox Systems Ltd for the studentship support for C. Bontozoglou.

Open Access This article is licensed under a Creative Commons Attribution 4.0 International License, which permits use, sharing, adaptation, distribution and reproduction in any medium or format, as long as you give appropriate credit to the original author(s) and the source, provide a link to the Creative Commons licence, and indicate if changes were made. The images or other third party material in this article are included in the article's Creative Commons licence, unless indicated otherwise in a credit line to the material. If material is not included in the article's Creative Commons licence and your intended use is not permitted by statutory regulation or exceeds the permitted use, you will need to obtain permission 
directly from the copyright holder. To view a copy of this licence, visit http://creativecommons.org/licen ses/by/4.0/.

\section{References}

1. R. Furstenberg, C.A. Kendziora, J. Stepnowski, S.V. Stepnowski, M. Rake, M.R. Papantonakis, V. Nguyen, G.K. Hubler, R.A. McGill, Stand-off detection of trace explosives via resonant infrared photothermal imaging. Appl. Phys. Lett. 93, 224103 (2008). https://doi.org/10.1063/1.3027461

2. C.A. Kendziora, Robert M. Jones, R. Furstenberg, M. Papantonakis, V. Nguyen, R.A. McGill, Infrared photothermal imaging for standoff detection applications, Proc. SPIE 8373, p. 83732H, (2012). https://doi.org/10.1117/12.918594

3. C.A. Kendziora, R. Furstenberg, M. Papantonakis, V. Nguyen, J. Borchert, J. Byers, R.A. McGill, Infrared photothermal imaging of trace explosives on relevant substrates. Proc. SPIE 8709, 87090O (2013). https://doi.org/10.1117/12.2015970

4. G. Rasskazov, A. Ryabtsev, M. Dantus, Eye-safe near-infrared trace explosives detection and imaging. Opt. Express 25, 5832-5840 (2017). https://doi.org/10.1364/OE.25.005832

5. R.D. Julia, J.M. William, Quantum-cascade-laser-based detector for proximal screening of surface contaminants, 15 August 2016, SPIE Newsroom. https://doi.org/10.1117/2.1201607.006642

6. R.H. Farahi, A. Passian, L. Tetard, T. Thundat, Pump-probe photothermal spectroscopy using quantum cascade lasers. J. Phys. D Appl. Phys. 45, 125101 (2012). https://doi.org/10.1088/00223727/45/12/125101

7. R.E. Imhof, D.J.S. Birch, F.R. Thornley, J.R. Gilchrist, T.A. Strivens, Opto-thermal transient emission radiometry. J. Phys. E: Sci. Instrum. 17, 521-525 (1984)

8. R.E. Imhof, A.D. McKendrick, P. Xiao, Thermal emission decay Fourier transform infrared spectroscopy. Rev. Sci. Instrum. 66, 5203-5213 (1995)

9. P. Xiao, Photothermal radiometry for skin research. Cosmetics 3, 10 (2016). https://doi.org/10.3390/ cosmetics 3010010

10. P. Xiao, J.A. Cowen, R.E. Imhof, In-vivo transdermal drug diffusion depth profiling-a new approach to opto-thermal signal analysis. Anal. Sci. 17, s349-s352 (2001). https://doi.org/10.14891/ analscisp.17icpp.0.s349.0

11. P. Xiao, X. Ou, L.I. Ciortea, E.P. Berg, R.E. Imhof, In-vivo skin solvent penetration measurements using opto-thermal radiometry and fingerprint sensor. Int. J. Thermophys. 33, 1787-1794 (2012). https://doi.org/10.1007/s10765-012-1318-6

12. P. Xiao, R.E. Imhof, Opto-thermal measurement of water distribution within the stratum corneum, in Skin Bioengineering Techniques and Applications in Dermatology and Cosmetology, vol. 26, Current Problems in Dermatology, ed. by P. Elsner, A.O. Barel, E. Berardesca, B. Gabard, J. Serup (Karger, Basel, 1998), pp. 48-60

Publisher's Note Springer Nature remains neutral with regard to jurisdictional claims in published maps and institutional affiliations. 